\title{
La protección jurídica del derecho humano a la educación y su proyección en el ámbito internacional
}

\author{
Mariana Blengio Valdés \\ Doctora en Derecho y Ciencias Sociales pela UDELAR, Profesora \\ en la Universidad de la República, Montevideo, Uruguay \\ ius@netgate.com.uy
}

\begin{abstract}
Resumen El derecho a la educación se encuentra en la actualidad ampliamente reconocido en las fuentes de derecho interno yderecho internacional de los derechos humanos. Su consagración normativa se observa en dos ámbitos. En primer lugar la incorporación del derecho humano a la educación en las constituciones nacionales, lo que le otorga el máximo nivel de jerarquía normativa, previéndose en diferentes cartasespecificaciones que aluden a singularidades de cada pueblo o región. En segundo lugar a través de la elaboración y aprobación en el seno de las organizaciones internacionales como la ONU y la OEA, de una larga nómina de instrumentos con diferentes efectos jurídicos que reconocen el derecho humano a la educación. Estos documentos apuestan además, a profundizar elcontenido de la educación, al resaltar la relevancia de promover el respeto a los derechos humanos como elemento particular a atribuir a los procesos educativos a nivel formal y no formal en todos los nivelesy áreas de la enseñanza.

La incorporación del contenido a atribuir a la educación en el marco de la promoción de los derechos humanos elemento constante de tales instrumentos, ha contribuido a la introducciónen los programas educativos de aspectos vinculados a los valores humanos así como también al desarrollo demetodologías acordes con tal objetivo. Esteenfoque ha logrado integrar estaperspectiva en la educación,como una importante herramienta para potenciar el proceso de formación de los ciudadanos, y como forma de contribuir ala toma de conciencia de los derechos y libertades, así como la prevención y erradicación de conductas ilegítimas y violentas que menoscaban la convivencia humana en el marco del Estado de Derecho.
\end{abstract}

Palabras clave: educación, derechos humanos, fuentes, Estado de Derecho.

\section{Introducción}

I a educación como derecho humano ha sido reconocida paulatiLnamente por el ordenamiento jurídico de los diferentes estados e incorporada a texto expreso al conjunto de normas vigentes.

Por su parte la comunidad internacional ha incluido este derecho en documentos de diferente naturaleza jurídica como ser declaraciones y tratados. En la actualidad el derecho a la educación se erige para gran parte de la doctrina, como una norma de "ius cogens" o "norma imperativa de derecho internacional". Se entiende por tales aquellas que "sean aceptadas o reconocidas por la comunidad internacional en su conjunto como una norma que no admite acuerdo en contrario". ${ }^{1}$ En

1. Convención de Viena sobre Derecho de los Tratados entre Estados. Artículo 53. Disponible en: http://www.derechos.org/nizkor/ley/viena.html Fecha de consulta: 1.8.2013. 
definitiva son aquellas normas que según se formula, "por la naturaleza de su objeto, la importancia de su contenido y su aceptación universal no admiten pacto en contrario, el que se considerará nulo". ${ }^{2}$

Partiendo de la base de la coexistencia de fuentes nacionales e internacionales que refieren en forma directa al derecho a la educación destacaremos algunos aspectos en relación al proceso de positivación.

\section{Derecho humano a la educación en el derecho interno}

El proceso de incorporación de la educación como derecho humano en los diferentes ordenamientos jurídicos se proyecta paulatinamente a partir del siglo XX. Su inclusión contiene diferentes matices y se vincula en muchos casos a las particularidades de cada pueblo. Por lo general su positivación opera en el máximo nivel de jerarquía, es decir en las constituciones de los estados. Así pueden verse las cartas fundamentales de distintos países americanos dondese incluye la educación como un derecho de la persona. Ello sin perjuicio de las regulaciones legales que reglamentan o profundizan el alcance del derecho. ${ }^{3}$

Del estudio de las diferentes constituciones americanas podemos puntualizar algunos aspectos en común:

a) La educación como derecho humano y por tanto imprescindible para lograr el desarrollo integral de la persona y su mejor comprensión e integración a la sociedad;

b) La responsabilidad conjunta de los padres y del Estado en el proceso educativo de los menores de edad;

c) El derecho de los padres a elegir la educación de sus hijos sin perjuicio de la autonomía y libertad de los niños y adolescentes;

d) El reconocimiento de la diversidad cultural y las tradiciones culturales como elementos esenciales que proyectan el valor en si del proceso educativo;

e) La educación a lo largo de toda la vida.

A este punteo podemos agregar otros aspectos de la temática que han sido incluidos como normas constitucionales. Entre ellos: gratuidad de la enseñanza primaria y media;libertad de enseñanza y de cátedra; laicidad. En forma más reciente: la educación inicial;la formación de los adultos; la educación física y la eliminación de las barreras de acceso al conocimiento para las personas con capacidades diferentes.

Así también las tradiciones culturales de los pueblos nativos y el necesario respeto a la diversidad tanto en relación a costumbres como expresiones orales. Resulta ilustrativa en tal sentido la Constitución del Paraguay (1992). Su Capítulo VII titulado "De la educación y la cultura" incluye 12 artículos que transitan sobre diversos aspectos a destacar: fines de la educación; derecho de aprender y libertad de enseñar; responsabilidad educativa; obligaciones del estado; enseñanza en lengua materna; educación técnica; universidad; fondos para becas; patrimonio cultural; promoción de los deportes así como también aspectos vinculados al presupuesto del estado en esta área. ${ }^{4}$

El artículo 73 establece: "Toda persona tiene derecho a la educación integral y permanente, que como sistema y proceso se realiza en el contexto de la cultura de la comunidad. Sus fines son el desarrollo pleno de la personalidad humana y la promoción de la libertad y la paz, la justicia social, la solidaridad, la cooperación y la integración de los pueblos; el respeto a los derechos humanos y los principios democráticos; la afirmación del compromiso con la patria, de la identidad cultural y la formación intelectual moral y cívica, así como la eliminación de los contenidos educativos de carácter discriminatorio. La erradicación del analfabetismo y la capacitación para el trabajo son objetivos permanentes del sistema educativo".

El análisis de este capítulo de la Constitución del Paraguay nos permite ilustrar el nexo significativo entre educación y cultura. Así también la responsabilidad de promover la educación como un cometido esencial que se enmarca dentro de los fines del propio estado. Por su parte la diversidad cultural se pone de manifiesto al reconocerse en el artículo 77 la enseñanza en los comienzos del proceso escolar en la lengua oficial materna del educando sin perjuicio de instruirse en el conocimiento y empleo de ambos idiomas oficiales de la República: "La enseñanza en los comienzos del proceso escolar se realizará en la lengua oficial materna del educando. Se instruirá asimismo en el conocimiento y en el empleo de ambos idiomas oficiales de la República. En el caso de las minorías étnicas cuya lengua materna no sea el guaraní, se podrá elegir uno de los dos idiomas oficiales".

En forma significativa también la Constitución del Paraguay introduce la promoción de los deportes especialmente los de carácter no profesional, estimu-

2. Pastori, Alejandro. "Las fuentes y normas del Derecho Internacional Público ante la fragmentación de la materia". En Revista de Derecho Público n. 43, editada por FCU, Montevideo, 2008, p. 143.

3. Blengio Valdes, Mariana y Gros Espiell, Héctor. "El derecho a la educación en las Constituciones de América Latina y el Caribe". Revista de Ciencias Jurídicas de la Universidad de las Palmas de Gran Canaria, n. 8 e 9, Las Palmas de Gran Canaria, 2003, p. 75 a 103.

4. Constitución de la República del Paraguay sancionada y promulgada el 20 de junio de 1992, Capítulo VII artículos 73 a 85 . Disponible en: http://www.snna.gov.py/inicio/index.php/institucional/marco-normativo/item/168-constituci\%C3\%B3n-nacional-de-la-rep\%C3\%BAblica-delparaguay. Fecha de consulta: 26 agosto 2013. 
lando con ello la educación física (artículo 84). ${ }^{5}$ Este aspecto resulta para algunas sociedades un elemento que aún no ha adquirido la importancia que el mismo tiene en relación al desarrollo integral del individuo.

\section{El derecho humano a la educación en el derecho internacional de los derechos humanos}

En el plano internacional documentos de especial trascendencia han incorporado el derecho a la educación como un compromiso de los estados en relación a las personas que habitan en su jurisdicción.

En forma cronológica tomando como punto de partida el fin de la Segunda Guerra Mundial la Declaración Universal de Derechos Humanos adoptada el 10 de diciembre de 1948 por la Asamblea General de la Organización de las Naciones Unidas ${ }^{6}$ consagró en su artículo 26 una amplia referencia a la educación. Así luego de expresar que "toda persona tiene derecho a la educación" señala la necesidad de que sea gratuita por lo menos en la instrucción fundamental y elemental así como también generalizada en otros niveles.

Este artículo prevé en su inciso 2 en relación al objeto mismo de la educación, que la misma apostará al "pleno desarrollo de la personalidad humana y el fortalecimiento del respeto a los derechos humanos y a las libertades fundamentales, favorecerá la comprensión, la tolerancia y la amistad entre todas las naciones y todos los grupos étnicos o religiosos"?

La materia educativa fue encarada en forma específica por la Unesco ${ }^{8}$ fundada el 16 de noviembre de 1945 como agencia especializada del sistema de las Naciones Unidas. En la Constitución de la UNESCO que entró en vigor el 4 de noviembre de 1946 se concibe a la educación como uno de los medios para contribuir a la paz. Entre sus objetivos se encuentra la erradicación de la pobreza, el desarrollo sostenible y el diálogo intercultural, la promoción de las ciencias y la cultura, la comunicación y la información. El Preámbulo de la Constitución de la UNESCO ex- presa ilustrativamente lo siguiente: “... puesto que las guerras nacen en la mente de los hombres, es en la mente de los hombres donde deben erigirse los baluartes de la paz".

La labor de la UNESCO ha sido constante a lo largo de más de medio siglo. Paulatinamente la organización que cuenta en la actualidad con 195 miembros ha ido adoptando tratados de especial relevancia en áreas que vinculan a la educación y a la cultura como ser la no discriminación en la enseñanza, los derechos de autor, las ciencias exactas, la promoción de la educación, la bioética, la diversidad cultural, el patrimonio mundial y más recientemente el deporte entre otros aspectos de trascendente actualidad. Los estados han incorporado dichos tratados a sus diferentes ordenamientos y en virtud de los mecanismos establecidos en los textos constitucionales para proceder a ello.

Capítulo especial lo merecen los nuevos desarrollos que la reflexión en torno a la ética en la biología ha generado a partir de las últimas décadas del siglo $\mathrm{XX}$ en el mundo entero. Aquí también la UNESCO protagoniza un liderazgo en tanto apuesta al equilibro entre la libertad y los derechos con el fin de asegurar el respeto y reconocimiento de la dignidad del ser humano. Ejemplos de esta labor lo ofrecen la trilogía de documentos universales adoptados por la organización: Declaración sobre el Genoma y los Derechos Humanos en el año 1997; Declaración Internacional sobre Datos Genéticos Humanos del 2003; y la Declaración sobre Bioética y Derechos Humanos de 2005. ${ }^{9}$ El principio de reconocimiento de la dignidad humana se proyecta entonces desde esta reflexión teórica a la práctica de la ciencia. Lo que ha determinado la introducción en la agenda política de la racionalización del poder científico.

El derecho a la educación también ha sido incluido en otros tratados suscritos en el ámbito universal. A modo de ejemplo podemos mencionar el Pacto de Derechos Económicos Sociales y Culturales aprobado en el año $1966 .{ }^{10} \mathrm{Su}$ artículo 13 establece que los estados partes "reconocen el derecho de toda persona a la educación". Para luego continuar con los objetivos de la educación, los requisitos de obligatoriedad y gratuidad de la enseñanza primaria, la generalización de la educación en otros niveles incluidos el superior, la libertad

\footnotetext{
5. Constitución de la República del Paraguay. Artículo 84: "De la promoción de los Deportes. El Estado promoverá los deportes, en especial los de carácter no profesional, que estimulen la educación física, brindando apoyo económico y exenciones impositivas a establecerse en la ley. Igualmente, estimulará la participación nacional en competencias internacionales".
}

6. Disponible en: http://www.un.org/es/documents/udhr/Fecha de consulta: 12 agosto 2013.

7. Disponible en: http://www.un.org/es/documents/udhr/ Fecha de consulta: 26 agosto 2013.

8. Disponible en: http://www.unesco.org/new/es/unesco/about-us/who-we-are/history/constitution/ Fecha de consulta: 26 agosto 2013

9. Pueden consultarse en el sitio de la UNESCO disponible en: http://www.unesco.org/new/es/social-and-human-sciences/Fecha de consulta: 26 agosto 2013

10. Disponible en: http://www2.ohchr.org/spanish/law/cescr.htm Fecha de consulta: 12 agosto 2013. 
de los padres de elegir la escuela o centro educativo a la cual concurrirán sus hijos. Este reconocimiento del derecho a la educación constituye una obligación de los estados partes que convienen en adoptar las medidas necesarias destinadas a lograr progresivamente la plena efectividad de los derechos reconocidos en el Pacto tal cual lo establece su artículo 2.

En el marco de este tratado se establecen mecanismos internacionales de control internacional. En forma concreta con la creación del Comité de Derechos Económicos Sociales y Culturales, cuyo cometido radica en promover el cumplimiento por parte de los estados del referido pacto. ${ }^{11}$

La inclusión del derecho humano a la educación en el sentido que le da el Pacto del año 1966 vincula en forma general a todas las personas de la familia humana. En forma posterior otros tratados de derechos humanos que abordan problemáticas concretas o cuyo sujeto de derecho lo constituyen los integrantes de grupos vulnerables, además de reiterar el derecho humano a la educación lo refieren directamente a las características de la problemática o del sujeto que se protege. Ejemplo de ello lo constituye la Convención sobre la Eliminación de Todas las Formas de Discriminación de la Mujer aprobado por la Asamblea General de las Naciones Unidas en $1979 .{ }^{12}$ Su artículo 5 establece: "Los Estados Partes tomarán todas las medidas apropiadas para... b) Garantizar que la educación familiar incluya una comprensión adecuada de la maternidad como función social y el reconocimiento de la responsabilidad común de hombres y mujeres en cuanto a la educación y al desarrollo de sus hijos, en la inteligencia de que el interés de los hijos constituirá consideración primordial en todos los casos".

Por su parte la Convención de los Derechos del Niño ${ }^{13}$ dedica dos extensos artículos a la temática. En el número 28 parte del reconocimiento del niño a la educación y los aspectos generales reiterando muchos de los incluidos en el PDESC sin perjuicio de la particularidad que el colectivo niñez contiene. Entre estos: "Adoptar medidas para fomentar la asistencia regular a las escuelas y reducir las tasas de deserción escolar". Reviste especial interés para este estudio el artículo 29, donde se establece el compromiso de los estados partes de encaminar la educación en base a determinados parámetros que describe en forma pormenorizada en sus diferentes incisos. Entre ellos: "el desarrollo de la personalidad, las aptitudes y la capacidad mental y física; el respeto a los derechos humanos; el respeto de sus padres y de su propia identidad cultural; de su idioma y sus valores". Así también "preparar al niño para asumir una vida responsable en una sociedad libre, con espíritu de comprensión, de paz, tolerancia, igualdad de los sexos y amistad entre los pueblos, grupos étnicos, nacionales y religiosos y personas de origen indígena".

En el ámbito americano específicamente de la OEA la Declaración Americana de Derechos y Deberes del Hombre ${ }^{14}$ adoptada en 1948 estableció en su artículo XII este derecho. Posteriormente la Convención Americana sobre Derechos Humanos ${ }^{15}$ (artículos 12.4 y 26) incluye el derecho a la educación. En tanto el Protocolo Adicional a dicha Convención ${ }^{16}$ (Protocolo de San Salvador) aprobado en el año 1988 refiere concretamente al derecho humano a la educación y profundiza su alcance en el artículo 13 reforzando el mecanismo de control que a nivel interamericano asegure su efectividad.

Al igual que en el ámbito universal algunas convenciones americanas como la destinada a Prevenir, Sancionar y Erradicar la Violencia contra la Mujer ${ }^{17}$ (Convención de Belem do Pará) establecieron la necesidad de luchar contra los estereotipos y las prácticas basadas en razones injustificadas de desigualdad. Es ilustrativo al respecto referir al artículo 8 de la referida Convención el cual dispone la obligación de los estados de "modificar los patrones socioculturales de conducta de hombres y mujeres, incluyendo el diseño de programas de educación formales y no formales apropiados a todo nivel del proceso educativo, para contrarrestar los perjuicios y costumbre y todo otro tipo de prácticas que se basen en la premisa de la inferioridad o superioridad de cualquiera de los géneros o en los papeles estereotipados para el hombre y la mujer que legitiman o exacerban la violencia contra las mujeres".

Sería tedioso y no es el objeto de este texto identificar todas y cada una de las normas de fuente internacional que refieren a la educación. Sin embargo es de orden afirmar que su larga lista consolida lo afirmado al comienzo,en el sentido de considerar el derecho humano a la educación como una norma imperativa de derecho internacional no susceptible de pacto en contrario.

11. El Comité de DESC fue creado por Resolución 1985/17 de 28 de mayo de 1985 del ECOSOC.

12. Disponible en: http://www.un.org/womenwatch/daw/cedaw/text/sconvention.htm Fecha de consulta: 12 agosto 2013.

13. Disponible en: http://www2.ohchr.org/spanish/law/crc.htm Fecha de consulta: 12 agosto 2013

14. Disponible en: http://www.oas.org/es/cidh/mandato/Basicos/declaracion.asp Fecha de consulta: 12 agosto 2013.

15. Disponible en: http://www.oas.org/dil/esp/tratados_B-32_Convencion_Americana_sobre_Derechos_Humanos.htm Fecha de consulta: 12. agosto 2013.

16. Disponible en: http://www.oas.org/juridico/spanish/Tratados/a-52.html Fecha de consulta: 12 agosto 2013

17. Disponible en: http://www.oas.org/juridico/spanish/tratados/a-61.html Fecha de consulta: 12 agosto 2013 
Ahora bien, sin perjuicio de este basto reconocimiento normativo que como hemos visto trasciende las fronteras de cada estado, las cifras en materia de educación siguen demostrando un abismo entre la norma y la realidad. Según datos de la Unesco:

"En la actualidad, 776 millones de jóvenes y adultos, vale decir, el $16 \%$ de la población mundial adulta son analfabetos. Pese a los grandes avances logrados desde 2000, 75 millones de niños, de los cuales el 55\% son niñas, siguen sin tener acceso a la enseñanza primaria. Millones de niños abandonan la escuela sin haber aprendido las nociones elementales de lectura, escritura y aritmética". 18

Datos estadísticos por demás significativos cuyo encuadre a nivel de cada país americano ameritaría un capítulo aparte. En el Informe Regional elaborado en el año 2007 por la UNESCO sobre "Situación Educativa en América Latina y el Caribe. Garantizando la Educación de Calidad para Todos" emerge claramente la realidad americana en materia educativa. El documento pone en evidencia la dimensión de inequidad y dificultades no solamente en el acceso a la educación sino la permanencia en el sistema educativo y la eficacia misma de los programas de estudio que pretenden aplicarse. ${ }^{19}$ Además de otros problemas como la pobreza que inciden negativamente en el proceso de formación de los individuos.

Algunas conclusiones de este extenso documento resulta interesante destacar: ${ }^{20}$

a. La ampliación de la educación a la primera infancia como desafío tanto en relación a la importancia directa como en el impacto generacional;

b. La conclusión universal de la educación primaria en carácter de mínimo irrenunciable. Así se afirma que: "La atención de las necesidades educativas de jóvenes y adultos resulta un área de gran preocupación que demanda de esfuerzos mayores. Los servicios actuales son muy limitados o son un canal alternativo de formación para niños que trabajan; lo que no puede pasar inadvertido";

c. La imperiosa reducción del analfabetismo a través de programas de educación para adultos entre otras medidas;

d. Asegurar el aprendizaje de contenidos mínimos e imprescindibles;

El informe establece que: "Las brechas de equidad identificadas representan un desafío particular- mente crítico porque compromete seriamente las posibilidades de garantía del derecho a la educación. Las capacidades de los sistemas educativos para dejar de reproducir las desigualdades sociales es un elemento crítico de su desempeño sobre el cual es preciso trabajar con mayor intensidad".

\section{La educación en derechos humanos}

La educación en derechos humanos como proceso educativo integrador se ha ido proyectando en los países americanos sin perjuicio de muchas otras iniciativas que podrían referirse en otros continentes como el europeo.

Está basada en una dimensión humanista de la educación y cuenta con exponentes de especial relevancia intelectual en este campo que han fundamentado esta perspectiva integradora del proceso educativo. En forma temprana Paulo Freire fundamenta esta mirada al expresar:

“E neste sentido que ensinar não é ação pela qual um sujeito criador dá forma, estilo ou alma a um corpo indeciso e acomodado. Não há docência sem discência, as duas se explicam e seus sujeitos apesar das diferenças que os conotam, não se reduzem à condição de objeto, um de outro. Quem ensina aprende ao ensinar e quem aprende ensina ao aprender". ${ }^{21}$

Estos enfoques permitieron desarrollar un campo nuevo en la educación que se basa en la incorporación y trasmisión de valores enmarcados en el proceso de "aprehender" la realidad y no visualizarla como un campo teórico ajeno a nuestra vida.

La educación en derechos humanos supone la proyección de una cultura capaz de incorporar en la mente y en el accionar de las personas los principios que hacen a su esencia humana entre los cuales la autonomía constituye un rasgo esencial. El ser autónomo e informado se presenta como un interlocutor que podrá asumir las decisiones en forma individual y consciente.

En el desarrollo de esta perspectiva educativa que se asienta en las particularidades de cada sociedad, también incide el fuerte impulso ha promovido desde las organizaciones internacionales. Así es por demás

18. Disponible en: http://unesdoc.unesco.org/images/0014/001473/147330s.pdf. Fecha de consulta: 26 de agosto 2013.

19. Puede ampliarse en el Informe regional de revisión y evaluación del progreso de América Latina y el Caribe hacia la educación para todos en el marco del proyecto Regional de Educación (EPT/PRELAC) 2007. Disponible en:http://unesdoc.unesco.org/images/0015/001528/152894s. pdf. Fecha de consulta: 26 agosto 2013.

20. Ob cit nota anterior pp. 21 y 22

21. Freire, Paulo. Pedagogia da autonomia. Editada por Paz e Terra, Brasil, 1996, p. 23. 
ilustrativa la Resolución 49/184 de la Asamblea General de las Naciones Unidas en la cual se proclamó el Decenio de las Naciones Unidas para la educación en la esfera de los derechos humanos (1995-2004). Entre los aspectos relevantes del Plan de Acción estructurado por la organización se encuentran dos que específicamente interesan a las Universidades y demás centros educativos: la creación y fortalecimiento de programas para la educación en la esfera de los derechos humanos y la preparación de material didáctico.

En forma consecuente con tales acciones la Asamblea General ha ideado las denominadas "Directrices para la elaboración de planes nacionales de acción para la educación en la esfera de los derechos humanos". En tal documento la organización señala que la educación en pro de los derechos "es fundamental y puede contribuir a la disminución de violaciones de derechos humanos y a la creación de sociedades libres, justas y pacíficas". ${ }^{22}$

Varios países siguiendo las directrices de la organización han ideado Planes Nacionales de Derechos Humanos. En su instrumentación que año a año va dando sus frutos han encontrado múltiples tropiezos sin perjuicio de lo cual entendemos que las acciones que promuevan esta perspectiva integradora son por demás positivas. Puede verse en relación al contenido dado a dichos planes el ejemplo de Brasil, país que lanzó en el año 2003 un "Plano Nacional de Educação em Direitos Humanos". En dicho documento se expresa que:

"A democracia, entendida como regime alicerçado na soberania popular, na justiça social e no respeito integral aos direitos humanos, é fundamental para o reconhecimento, a ampliação e a concretização dos direitos. Para o exercício da cidadania democrática, a educação, como direito de todos e dever do Estado e da família, requer a formação dos(as) cidadãos(as)" ${ }^{23}$

Las iniciativas en materia de educación en derechos humanos comienzan a desarrollarse a fines del siglo XX en América Latina ya no como eventos o actividades aisladas y puntuales en los cuales se abordan problemáticas vinculadas a los valores y los derechos sino como propuestas educativas destinadas a formar a los estudiantes con una perspectiva de tolerancia, solidaridad y fraternidad en la futura misión profesional. Esta nueva tendencia se opone a la visión autoritaria y subordinada del proceso educativo. Lo cual incluye también el desarrollo de metodologías integradoras acordes con los presupuestos planteados.
El desarrollo de metodologías aplicables en las diferentes áreas, ha llevado a la conformación de principios o pautas rectoras de la formación en derechos humanos. Entre estos podríamos destacar algunos esenciales como el que vincula al ámbito en el cual se desarrolla la actividad. Atribuir al proceso educativo un proceso lógico y coherente entre el "decir" y el "hacer". En tal sentido las estrategias didácticas que lleve adelante el docente en el aula serán claramente significativas del ejercicio armónico del modelo que se persigue. En un ámbito autoritario y descalificador del educando, difícilmente se logre consolidar el proceso educativo desde la integración de los valores humanos.

Son varios los principios que se sugieren como rectores de las prácticas de la educación crítica que promueve la educación en derechos humanos y cuyo desarrollo excede este abordaje.

A modo de conclusión y sin perjuicio de remitirnos a los interesantes y valiosos estudios que en materia de educación en derechos humanos se vienen desarrollando a partir de la práctica y la investigación de múltiples educadores, destacamos la existencia de un vínculo ineludible entre la educación como derecho humano y la educación en derechos humanos.

El derecho humano a la educación obliga a educar en derechos humanos. Y por ende son necesarias las perspectivas que promueven la eficacia del derecho humano a la educación partiendo de esa perspectiva integradora del proceso educativo.

\section{Vínculo entre educación y estado de derecho}

Es el Estado de Derecho el marco indispensable para garantizar el derecho humano a la educación. El vínculo existente entre la educación y Estado de Derecho resulta de especial interés. Ello en tanto muchos de los contenidos que se indican como prioritarios para la educación en derechos humanos refieren a la importancia de comprender el Estado de Derecho como el sustento imprescindible para hacer valer los mismos.

Del punto de vista del formador en derechos humanos esta afirmación comprende una doble labor. Por un lado la comprensión del concepto del Estado de Derecho, su significado, características y proyección. Y por otro, la necesidad de que este concepto sea fácilmente trasmitido a los grupos o colectivos para que incorporen su significado y lo asuman como

22. Asamblea General de la ONU. A/52/469/Add.1. 20 denoviembre de 1997. Quincuagésimo segundo período de sesiones. Cuestiones relativas a los derechos humanos incluidos distintos criterios para mejorar el goce efectivo de los derechos humanos y las libertades fundamentales. Directrices para la elaboración de planes nacionales para la educación en la esfera de los derechos humanos.

23. Plano Nacional de Educaçao em Direitos Humanos. Secretaria Especial dos Direitos Humanos., Presidencia de la República, Ministerio de Educaçao, Unesco, Brasil, 2007, p. 17. 
"actitud" o "acción" y no solamente como "concepto teórico". Para que pase de ser una lectura pasiva de la realidad y se constituya como un elemento que lo singulariza en el contexto educativo.

Se transforma en prioritaria esta comprensión si nos detenemos en determinados colectivos especialmente vinculados a la esencia misma del estado como pueden ser las fuerzas del orden, la seguridad o la policía. Trabajar conceptos como Estado de Derecho y principios que lo regulan,se transforma en una tarea trascendente a incorporar en el proceso de formación policial. El policía en su carácter de servidor público convive con jóvenes y adultos, hombres y mujeres en una infinidad de situaciones que lo interpelan día a día.

Resulta ilustrativo referir aquí a experiencias mantenidas en formación de policías en Uruguay. Desde hace ya algunos años se ha incorporado la materia específica "Derechos Humanos" a la capacitación del policía. Así también se imparte un curso sobre la temática "Violencia doméstica".

\section{1. ¿Es posible educar a un policía en derechos humanos?}

La respuesta es afirmativa y muchas experiencias ya instrumentadas en diversos países lo demuestran. La educación de los servidores públicos del orden en todos los casos debe incluir la formación en derechos humanos. De modo de trasmitir al policía la importancia de la temática. No solamente para que aprenda contenidos teóricos sino para que incorpore dichos contenidos en su accionar. Y con ello la humanización de su labor marque como nota característica su actuación.

Múltiples problemas se presentan al momento de la capacitación de este grupo de trabajadores. Algunos formales como por ejemplo quien es el formador más adecuado o recomendable para impartir la instrucción: ¿un civil o un agente policial?

Entendemos que sería reducir el contenido de esta pregunta limitarla a esta falsa oposición. La mejor educación en derechos humanos la impartirá quien tenga una formación en contenidos y aplique metodologías que promuevan esa perspectiva educadora en el ámbito policial. Es decir formadores capacitados en Educación en Derechos Humanos que puedan instrumentar los principios pedagógicos que aprendieron e incorporaron en su especialidad para atender las particularidades de este colectivo. De esta manera seguramente la labor se exitosa y permita abrir al policía al universo del respeto por los derechos humanos del cual él forma parte y no es un mero observador.

Por lo general la distancia que separa al educador en derechos humanos y a los policías se basa en el sentimiento de no pertenencia. Esto implica que el policía a quien se le capacita puede llegar a considerar que no posee derechos humanos. Que los denominados "derechos humanos" son propiedad del grupo que justamente él debe atender o "reprimir". En consecuencia la primera labor a realizar en caso de impartir enseñanza a la policía radica en generar la "conciencia de pertenencia". Es decir, la convicción de que son sus derechos los que también se protegen porque en realidad, son todos los derechos de todos los individuos los que deben asegurarse sin distinción del rol que ocupemos en la sociedad.

Concluimos entonces que la educación en el marco del Estado de Derecho supone la necesaria preocupación por impartir no solo a civiles la educación con contenido humanista y pacifista. Sino también aquellos que conviven con la violencia o la ilegalidad. Y que no por ello deberán ser violentos o realizar actos ilegítimos.

\section{El derecho de los derechos humanos y sus principios}

El Derecho de los Derechos Humanos como disciplina autónoma se funda en diferentes principios que le otorgan características singulares. Estos principios han sido consagrados en normas de fuente interna e internacional.

En el universo de contenidos de la educación en derechos humanos especial lugar ocupan los principios generales. Verdaderos pilares que aseguran la solidez intelectual de la tarea formativa en el marco del respeto a sus derechos. Pueden enumerarse entre ellos: a) el reconocimiento de la dignidad humana; b) in dubio pro persona; c) la igualdad; d) legalidad, entre otros. ${ }^{24} \mathrm{La}$ enseñanza de los principios que informan el derecho de los derechos humanos constituye una labor imprescindible que asegura un entendimiento genérico de la materia y permite al educador luego interiorizarse en aspectos específicos de la enseñanza.

A modo de ejemplo las sociedades del siglo XXImuestran un proceso de profundización de la consagración normativa tendiente a lograr la igualdad real también en aspectos tales como la intimidad o privacidad de las personas. De esta forma la ley ha comenzado a incorporar y proteger condiciones humanas invisibles hasta el momento sobre todo en relación al reconocimiento jurídico. Así puede verse la reciente legislación de Uruguay sobre igualdad de las personas en el matrimonio. ${ }^{25}$ La nueva norma transforma sustancialmente la regulación jurídica de la institución del matrimonio lo que incide directa o 
indirectamente en un universo amplio de derechos, deberes y garantías no solamente en relación a las dos personas adultas que contraerán formalmente el vínculo, sino también a su descendencia. La modificación se fundamenta en la necesidad de dar igual tratamiento jurídico a través del instituto del matrimonio, a la unión de dos personas del mismo sexo. Se reafirma con ello que la orientación sexual es un rasgo existencial de los seres humanos. Por tanto no resulta legítimo que el estado distinga e inhabilite a las personas para unirse en matrimonio en razón de su sexualidad. En los hechos este impedimento contraviene la igualdad de los individuos en la ley pues establece una desigualdad relativa a una condición existencial de los humanos.

Esta incorporación legislativa no se produjo del día a la mañana. Es producto de un proceso paulatino que transitó por etapas tan lejanas como la lucha contra la esclavitud y el destierro de ideas que distinguían categorías de humanos negando a algunos su calidad de tales. Supuso también el reconocimiento de la igualdad en materia de derechos políticos primero entre hombres y luego en relación a las mujeres. A lo cual se unió la lucha por la igualdad en materia social y laboral en un camino que apuesta a ofrecer equidad en las relaciones de trabajo. Mojones de una historia tensa y progresiva, a la cual seguramente se le irán agregando más capítulos.

El reto consiste entonces en incorporar la esencia de la igualdad. E introducir al educando en un universo de diversidad que opte por una mirada múltiple del universo. A partir de la comprensión de la dimensión de la igualdad de la condición humana y su diversidad como ser vivo.

Para concluir podemos señalar que la comprensión de los principios permite adentrarnos a problemáticas concretas facilitando la comprensión. Así el principio de igualdad basado en la esencia de la persona nos permite comprender cabalmente la existencia de construcciones culturales o estereotipos que ejercen una nociva influencia en la sociedad, aislando, estigmatizando o multiplicando estructuras de poder autoritarias y lejanas al respeto de valores intrínsecos a la personalidad humana.

\section{3. ¿Es la educación un camino para la paz?}

La educación es un camino para lograr la paz. Esta idea ha sido ampliamente desarrollada en las últimas décadas. Pensadores y educadores de talla universal han concluido en sus aportes que la educación es un camino para proyectar a la persona desde el conocimiento. ${ }^{26}$ La labor constante de muchos grupos e individuos demuestran con claridad un compromiso inquebrantable con el proceso formativo como preocupación por la consolidación de la tolerancia.

Sin embargo múltiples ejemplos a través de la historia demuestran que algunas de las accion es más atroces que puedan verificarse en contraposición a los valores humanos provienen de la acción o el asesoramiento de personas con alto grado de formación y educación.

Esta constatación nos lleva a la interrogante formulada en el título. Evidentemente daremos una respuesta afirmativa a la pregunta. Pues entendemos que efectivamente es la educación un camino para la paz. Sin embargo no es la "educación" como la acumulación repetitiva de datos. Pues el problema no estaría resuelto si las estadísticas que emanan de los muchos informes que se realizan sobre el estado de la educación incluyendo acceso y demás aspectos, fueran revertidas.

La aproximación al universo de la educación promueve actitudes de comprensión del ser individual y social. Y ayuda a los individuos a desenvolverse con otra solvencia en su comunidad. Sin embargo con esto no basta. El desafío radica en la incorporación de los valores en la educación y su aprendizaje "corporal", vivencial y no meramente teórico.

Por tanto la educación es un camino para la paz sin lugar a dudas. La educación que se funda e incorpora no solo en el pensamiento sino en el espíritu y el accionar,los valores intangibles del ser humano. Pues parten del concepto de persona como ser viviente universal, cuya condición existencial exige la valoración de su integridad como ente único. La inclusión corporal del concepto trasmitida en la acción profesional y singular es entonces uno de los caminos para asegurar la paz. 


\title{
Referencias
}

BIDART CAMPOS, Germán. Teoría general de los Derechos Humanos. Buenos Aires: Astrea, 1991.

BOBBIO, Norberto. El tiempo de los derechos. Madrid: Sistema, 1991.

CAGNONI, José Aníbal. El Estado de Derecho en el Derecho Internacional y el Derecho Interno. Montevideo: JAC, 2009.

CASADO, María. Sobre la educación en Bioética. Revista de Derecho Público, n. 39, Montevideo, 2011.

FREIRE, Paulo. Pedagogía da autonomía. São Paulo: Paz e Terra, 2004.

FERRAJOLI, Luigi. Derechos y garantías. España: Trotta, 1991.
MENEGUELLO CARDOSO, Clodoaldo. Libertade e igualdade nos direitos humanos. Educação em direitos humanos na formação universitária. Brasil: Cultura Acadêmica, 2009.

GIALDINO, Ronaldo. Derecho Internacional de los Derechos Humanos: principios, fuentes, interpretación y obligaciones. Buenos Aires: Abeledo Perrot, 2013.

PEREZ AGUIRRE, Luis. Si, digo Derechos Humanos. Montevideo: SERPAJ, 1991.

La educación en Derechos Humanos ante los desafíos del siglo XXI. Montevideo: Cátedra Unesco, 2010 .

\section{A proteção jurídica do direito humano e da educação e sua proteção no âmbito internacional}

\section{Resumo}

O direito e a educação se encontram na atualidade amplamente reconhecidos no ordenamento nacional e internacional dos direitos humanos. Sua consagração normativa se observa em dois âmbitos. Em primeiro lugar, na incorporação do direito humano à educação nas constituições nacionais, o que lhe outorga o máximo nível de hierarquia normativa, prevendo-se, em diferentes cartas, especificações que marcam singularidades de cada povo ou região. Em segundo lugar, por meio da elaboração e aprovação no seio das organizações internacionais, tais como a ONU e a OEA, de uma grande lista de instrumentos com diferentes efeitos jurídicos que reconhecem o direito humano à educação. Estes documentos buscam aprofundar o conteúdo da educação, principalmente ao ressaltar a importância de se promover o respeito aos direitos humanos como elemento particular atribuído aos processos educativos, em nível formal e não formal, em todos os níveis e áreas do ensino. A incorporação do conteúdo a ser atribuído à educação, no marco da promoção dos direitos humanos - elemento constante de tais instrumentos - vem contribuindo com a introdução, nos programas educativos, de aspectos vinculados tanto a valores humanos quanto ao desenvolvimento de metodologias concernentes com tal objetivo. Este enfoque tem conseguido integrar o processo de formação de cidadãos, como forma de contribuir com a tomada de consciência dos direitos e liberdades, com a prevenção e erradicação de condutas ilegítimas e violentas que desprezam a convivência humana no marco do Estado de Direito.

Palavras-chave: educação, direitos humanos, fontes do direito, estado de direito.

\section{Legal protection of the human right to education and its influence in international sphere}

\begin{abstract}
Currently, the right to education is widely recognized within sources of national and international human rights law. Its inclusion in Currently, the right to education is widely recognized within sources of national and international human rights law. Its inclusion in regulatory policy is twofold. In the first place, the human right to education, incorporated into national constitutions, grants it high regulatory importance. Different constitutions contain different provisions relating to distinct peoples and regions. Secondly, numerous documents developed and approved within international organizations such as the UN and the OAS, and varying in their scope of legal implications, recognize the elementary human right to education. These documents also have the objective of strengthening the contents of educational curricula, thereby highlighting the importance of promoting the respect for human rights as a particular
\end{abstract}


element of formal and informal educational processes at all levels and areas of education. The incorporation of educational content promoting human rights has contributed to the introduction of human values related curricula in educational programs and to the development of methodologies consistent with such goals. This approach has succeeded in integrating human rights perspectives into education, as an important tool for enhancing the process of civic education, and as a way to raise awareness about rights and freedom, as well as prevent and eradicate illegitimate and violent behaviors that undermine human coexistence within the framework of the rule of law.

Key words: education, human rights, sources, rule of law.

Data de recebimento do artigo: 13/5/2013

Data de aprovação do artigo: 20/12/2013 